\title{
Optical Constants of $\mathrm{CuO}$ and $\mathrm{ZnO}$ Particles in the Terahertz Frequency Range
}

\author{
Jaume Calvo-de la Rosa, ${ }^{1}$ Alexandre Locquet, ${ }^{2,3}$ Denis Bouscaud, ${ }^{4}$ Sophie Berveiller, ${ }^{4}$ and D.S. Citrin ${ }^{2,3}$ \\ ${ }^{1}$ Department of Materials Science and Physical Chemistry, Chemistry Faculty, Universitat de \\ Barcelona, Martí i Franquès 1, 08028 Barcelona, Spain \\ ${ }^{2}$ Georgia Tech-CNRS UMI2958, Georgia Tech Lorraine, 2 Rue Marconi, 57070 Metz, France \\ ${ }^{3}$ School of Electrical and Computer Engineering, Georgia Institute of Technology, Atlanta, Georgia \\ 30332-0250, USA \\ ${ }^{4}$ Laboratoire d'Etude des Microstructures et de Mécanique des Matériaux LEM3 (UMR CNRS 7239), \\ Arts et Métiers ParisTech, Université de Lorraine, 4 rue Augustin Fresnel, 57078 Metz, France
}

\begin{abstract}
The optical constants (complex dielectric function) of isotropically distributed $\mathrm{CuO}$ and $\mathrm{ZnO}$ particles in polyethylene pellets are measured by terahertz time-domain spectroscopy. The determination of the dielectric properties of these materials is of interest for energy-storage applications. Maxwell-Garnett theory is used to extract the contribution to the frequency-dependent optical constants of the oxide powders. The validity of the assumptions of Maxwell-Garnett theory are experimentally verified and self-consistency of the results of the model confirmed. On this basis, experimental complex permittivity values for isotropic $\mathrm{CuO}$ and $\mathrm{ZnO}$ oxide powders are reported in the $100 \mathrm{GHz}-3 \mathrm{THz}$ range.
\end{abstract}

\section{Introduction}

Metal oxides comprise a large class of materials with numerous applications ranging from precursors for further chemical processes to advanced functional applications such as energy storage [1], gas sensors [2], pigments, and food packaging [3]. Moreover, recent published work shows the growing interest paid to the design of new materials obtained by mixing simple oxides, and forming more complex structures such as ferrites or perovskites. Nevertheless even relatively simple oxides, such as $\mathrm{CuO}$ or $\mathrm{ZnO}$, may experience a change of their properties when they are reduced to micro or nano dimensions, making them of interest for catalysis [4] and gas sensing [5-7]. Furthermore, there has been increasing interest paid to the use of these materials as additives to improve the energy-storage capacity in various advanced ceramics. There have been many studies for both, $\mathrm{CuO}$ [8-11] and $\mathrm{ZnO}$ [12-15] highlighting their potential for energy-storage applications. Clearly, the dielectric properties of these oxides are key parameters that determine the final energy-storage properties of the composite. It should also be noted that oxide powders are used as additives in otherwise THz-transparent plastics, and such materials are of use for $\mathrm{THz}$ components or are used in applications where $\mathrm{THz}$ nondestructive evaluation is under exploration. Urgency is thus building one several fronts to characterize the dielectric properties of oxide powders in the $\mathrm{THz}$ regime.

While the dielectric properties of such particles are of interest across the electromagnetic spectrum, characterization in the terahertz $(\mathrm{THz})$ frequency range, conventionally from $\sim 100$ $\mathrm{GHz}$ to $10 \mathrm{THz}$, is of interest for a number of emerging applications in nondestructive evaluation, process monitoring, sensing, and spectroscopy. Pulsed $\mathrm{THz}$ techniques in particular are gaining momentum [16,17]. The main idea is to interrogate a sample with a $\mathrm{THz}$ pulse and to study how the reflected, transmitted, refracted, or scattered pulse is 
modified with respect to the incident pulse after interacting with the sample. THz timedomain spectroscopy (THz-TDS) has been applied to a variety of materials to determine their linear optical constants, in particular the refractive index, absorption coefficient, complex permittivity, and frequency-dependent conductivity. Numerous works in this field have used this technique to characterize explosives [18,19], drugs [20], organic [21-23], ceramic [2426], and composite materials [27].

At the same time, it is important to note that measuring the optical constants of powders is not without difficulty of interpretation. While a number of methods have been developed, diffuse reflectance spectroscopy [28] is a commonly applied technique of growing importance. The geometry of this and related approaches, however, may be impractical at $\mathrm{THz}$ frequencies. As an alternative, one can attempt to measure the effective optical constants in a straightforward transmission experiment in a sample in which the powder of interest is of a very low fill factor dispersed within a well characterized matrix material. Provided the fill factor is sufficiently low, scattering and diffuse effects are minimized and for particle size much smaller than the optical wavelength - and assuming spherical particles-one can apply Maxwell-Garnet (MG) theory [29,30] and related approaches to extract the optical constants of the powder constituent. Another assumption of MG theory is that the wavelength is long compared with the interparticle spacing - a condition manifestly not met. Nonetheless, MG theory is this approach we adopt here as to some extent the assumptions appear to be met. Still, the values inferred for the powder may differ from those of the bulk material. As mentioned above, this may in part be due to modification of the optical constants linked to the micro or nanostructure, but may also be due in part to limited applicability of MG theory to a given sample.

In this article we present structural and $\mathrm{THz}$ characterization of two semiconductor oxide powders, $\mathrm{CuO}$ and $\mathrm{ZnO}$, dispersed in pressed polyethylene (PE) pellets. Chemical as well as particle size and shape characterization of the oxide powders is carried out in order to discuss the respective contributions to the $\mathrm{THz}$ measurement. The analysis provides the frequencydependent complex dielectric constant from $\sim 180 \mathrm{GHz}$ to $3 \mathrm{THz}$. We further discuss our results in the light of existing measurements.

\section{Theoretical framework}

In linear THz-TDS in transmission mode, a $\mathrm{THz}$ electromagnetic pulse is incident (the reference) on the sample and the time-dependent amplitude and phase of the transmitted pulse are measured after propagation through the material. For simplicity, we assume that the wavelengths in the usable $\mathrm{THz}$ bandwidth of the experiments are much longer than the particle sizes (though this might not always be the case at the high-frequency end of the band in this study), enabling us to treat the medium as having uniform effective optical constants. The various frequency components of the transmitted pulse will experience attenuation and phase shift depending on the sample properties. While the experiment is carried out in the time domain (TD), the signals are sampled and processed in the frequency domain (FD) via a discrete Fourier transform (DFT).

We begin by reviewing the relationships between various optical constants [31]. We shall only consider the linear optical properties and shall assume the bulk material constituents are isotropic, although this is manifestly not the case for $\mathrm{CuO}$ in particular. We discuss this simplification later in our study. After DFT, the transmittance $T(v)$ at frequency $v$ can be calculated by taking the ratio of the electric field $E_{S}$ at $v$ transmitted through the pellet and the electric field of the reference $E_{R}$ also at $v$. From this ratio, it is possible to extract the frequency-dependent relative amplitude $A(v)$ and phase $\phi(v)$ of the transmitted field, 


$$
T(v)=\frac{E_{S}(v)}{E_{R}(v)}=A(v) e^{i \phi(v)} .
$$

The refractive index $n(v)$ is calculated from the propagation delay related to the difference in phase suffered during transmission, while the absorption coefficient $\alpha(v)$ is also related to the reduction in amplitude,

$$
\begin{gathered}
n(v)=1+\frac{c}{2 \pi v} \phi(v), \\
\alpha(v)=-\frac{2}{d} \ln \left[A(v) \frac{(n(v)+1)^{2}}{4 n(v)}\right]
\end{gathered}
$$

where $c$ is the in-vacuo speed of light and $d$ is the sample thickness. Equation (3) accounts for the first (but not multiple) transmission and reflection and the front and back interfaces of the pellet, respectively. The absorption coefficient $\alpha$ can be easily linked with the extinction coefficient $\kappa$, which corresponds to the absolute value of the imaginary part $\boldsymbol{\kappa}$ of the complex refractive index $\boldsymbol{n}-\boldsymbol{i} \boldsymbol{\kappa}$,

$$
\kappa=\frac{\alpha \lambda_{0}}{4 \pi}
$$

In this equation, $\lambda_{0}$ is the wavelength in vacuum, i.e., $\lambda_{0}=c / v$. The complex permittivity $\hat{\boldsymbol{\varepsilon}}=\boldsymbol{\varepsilon}^{\prime}+\boldsymbol{i} \boldsymbol{\varepsilon}^{\prime \prime}$ can be calculated from $n$ and $\alpha$ by using Eqs. (5) and (6) below. The real part of the permittivity is related to the stored energy, while the imaginary part is related to attenuation:

$$
\begin{gathered}
\varepsilon^{\prime}=n^{2}-\kappa^{2}=n^{2}-\left(\frac{\alpha \lambda_{0}}{4 \pi}\right)^{2}, \\
\varepsilon^{\prime \prime}=2 n \kappa=\frac{\alpha n \lambda_{0}}{2 \pi} .
\end{gathered}
$$

Moreover, the electrical conductivity $\sigma$ can be calculated from the imaginary part of the permittivity by

$$
\sigma=2 \pi v \varepsilon^{\prime \prime}=2 \pi v \varepsilon_{0} \varepsilon_{r}^{\prime \prime}
$$

Here $\varepsilon_{0}$ is the vacuum permittivity $\left(8.85 \times 10^{-12} \mathrm{~F} / \mathrm{m}\right)$ and $\varepsilon_{r}$ "' the imaginary component of the relative permittivity $\left(\varepsilon_{r}=\varepsilon / \varepsilon_{0}\right)$. Due to the typically high attenuation that this family of materials usually presents, the powders have been dispersed into a THz-transparent polymeric matrix in order to permit significant overall transmission through the samples and thus a large signal-to-noise-ratio (SNR). For this reason, the properties deduced using the above equations do not directly represent the properties of the oxide itself, but rather the macroscopic properties of the combination of the polymer matrix and the oxide powder. As one may expect, the amount of oxide dispersed into the polymer affects the observed properties. With the aim of quantifying the global properties of a mixture of host and inclusion materials, various effective medium theories have been developed [32]. One of the most common approximations is MG theory, which assumes that the electric field within each particle is a combination of the external field and the field caused by other particles $[16,33,34]$. This approximation leads to 


$$
\frac{\varepsilon_{e f f}-\varepsilon_{h}}{\varepsilon_{e f f}+2 \varepsilon_{h}}=f_{v} \frac{\varepsilon_{i}-\varepsilon_{h}}{\varepsilon_{i}+2 \varepsilon_{h}},
$$

where $\varepsilon_{e f f}, \varepsilon_{h}$, and $\varepsilon_{i}$ represent the permittivity of the effective medium (measured mixture), the host material, and the inclusions (powder), respectively; $f_{v}$ is the volume filling factor, i.e., the volume ratio that the inclusions occupy.

Despite the fact that this model was developed for spherical particles, it will be assumed to be valid for any shape as long as the medium is electrodynamically isotropic [33] (see below). In addition, MG theory assumes a distribution of noninteracting particles, avoiding percolation effects. For this reason, it is an accurate approximation when working with low $f_{v}(<50 \%)$ samples, and the host and inclusions' volumes are not comparable. In cases with higher $f_{v}$, other theoretical approaches (such as Bruggeman) may be employed. Finally, scattering is not accounted for, so inclusions and the distances between them should be much smaller than the wavelengths within the THz pulse bandwidth $[34,35]$. With this in mind, MG theory is used here to infer the permittivity of the oxide inclusions.

\section{Experimental procedure and setup}

Both starting oxide powders were obtained from Inoxia Ltd. (Cranleigh, UK). Polyethelene (PE) powder, used as the matrix in which the oxide powder is dispersed and pressed into pellets, was Inducos 13/1 (particle size $<80 \mu \mathrm{m}$ ).

To fabricate the pellets, a 13-mm diameter evacuable pellet die from Specac was used. Each constituent, weighted by a microbalance, was manually mixed. The mixture was filled into the pellet die and the die was then placed between the plates of a compressive machine for compaction of the sample powder applying a force of about $25 \mathrm{kN}$.

Scanning electron microscopy (SEM) images were obtained with a JEOL JSM 7001F microscope. Pellets were fixed to the sample holder by means of a conductive carbon tape. Low accelerating voltages ( 1 and $5 \mathrm{kV}$ ) were used to observe the samples, because of their limited electrical conductivity. Electron micrographs were obtained from secondary electron emission, giving a topographic contrast.

Pellets with various oxide-powder filling factors $f_{\mathrm{v}}$ were prepared for each material according to the aforementioned procedure. As we work with MG theory, all pellets are in the low- $f_{v}$ regime $(<<50 \%)$. Table 1 shows the density $(\rho)$ and porosity $(P)$ of each fabricated pellet.

Table 1. Physical characteristics of all fabricated pellets.

\begin{tabular}{cccccc}
$\begin{array}{c}\text { Sample } \\
\text { Name }\end{array}$ & $\begin{array}{c}\text { Oxide } \\
\text { Inclusions }\end{array}$ & $\begin{array}{c}\text { Weight } \\
\text { Filling } \\
\text { Factor, } \\
\boldsymbol{f}_{\boldsymbol{w}}(\boldsymbol{\%})\end{array}$ & $\begin{array}{c}\text { Volume } \\
\text { Filling } \\
\text { Factor, } \\
\boldsymbol{f}_{\boldsymbol{v}}(\boldsymbol{\%})\end{array}$ & $\begin{array}{c}\text { Experimental } \\
\text { Density, } \\
\boldsymbol{\rho}\left(\mathbf{g} / \mathbf{c m}^{\mathbf{3}}\right)\end{array}$ & $\begin{array}{c}\text { Porosity, } \\
\boldsymbol{P}(\boldsymbol{\%})\end{array}$ \\
\hline PE_ref & None & 0.0 & 0.0 & 0.81 & 13.8 \\
ZnO_02.5 & $\mathrm{ZnO}$ & 2.5 & 0.4 & 0.82 & 16.0 \\
ZnO_05.0 & $\mathrm{ZnO}$ & 5.0 & 0.9 & 0.84 & 15.3
\end{tabular}




$\begin{array}{llcccc}\text { ZnO_10.0 } & \mathrm{ZnO} & 10.0 & 1.9 & 0.85 & 18.0 \\ \mathrm{ZnO} 20.0 & \mathrm{ZnO} & 20.0 & 4.1 & 0.93 & 19.1 \\ \mathrm{ZnO} 30.0 & \mathrm{ZnO} & 30.0 & 6.8 & 0.99 & 21.9 \\ \mathrm{ZnO} 40.0 & \mathrm{ZnO} & 40.0 & 10.2 & 1.09 & 23.7 \\ \mathrm{CuO}+02.5 & \mathrm{CuO} & 2.5 & 0.4 & 0.81 & 16.4 \\ \mathrm{CuO}+05.0 & \mathrm{CuO} & 5.0 & 0.8 & 0.83 & 16.6 \\ \mathrm{CuO}+07.5 & \mathrm{CuO} & 7.5 & 1.2 & 0.85 & 16.4 \\ \mathrm{CuO}+10.0 & \mathrm{CuO} & 10.0 & 1.7 & 0.87 & 16.7 \\ \mathrm{CuO} 20.0 & \mathrm{CuO} & 20.0 & 3.6 & 0.96 & 16.8 \\ \mathrm{CuO} 30.0 & \mathrm{CuO} & 30.0 & 6.1 & 1.06 & 17.4 \\ \mathrm{CuO} 40.0 & \mathrm{CuO} & 40.0 & 9.2 & 1.17 & 18.8\end{array}$

$P$ was calculated assuming densities of $0.95 \mathrm{~g} / \mathrm{cm}^{3}, 5.61 \mathrm{~g} / \mathrm{cm}^{3}$, and $6.31 \mathrm{~g} / \mathrm{cm}^{3}$ for $\mathrm{PE}, \mathrm{ZnO}$, and $\mathrm{CuO}$ respectively, and applying Eq. (9) [36].

$$
P(\%)=100\left(1-\frac{\rho_{\text {exp }}}{\rho_{\text {ideal }}}\right)=100\left(1-\frac{\rho_{\text {ex }}}{f_{v} \rho_{\text {incl }}+\left(1-f_{v}\right) \rho_{\text {host }}}\right)
$$

where $\rho_{\text {exp }}\left(\rho_{\text {ideal }}\right)$ is the real (ideal) density of the pellet, whereas $\rho_{\text {incl }}\left(\rho_{\text {host }}\right)$ represents the density of the inclusion (host) material in the pellet; $f_{w}$ is the weight filling factor, which is related to the volume filling factor $f_{v}$ by

$$
f_{v}=\frac{\frac{f_{W}}{\rho_{\text {incl }}}}{\frac{f_{W}}{\rho_{\text {incl }}}+\frac{1-f_{W}}{\rho_{\text {host }}}} .
$$

THz transmission data through the pellets were obtained using a TeraView TPS Spectra 3000 in transmission mode. The experimental bandwidth (power spectrum of the THz pulses) extends from $\sim 180 \mathrm{GHz}$ to $3 \mathrm{THz}$. Before starting the measurements, samples were placed in a sample holder and the entire working area was purged with $\mathrm{N}_{2}$ for 10 minutes at 5 liters per minute to avoid water-vapor absorption lines obscuring the spectra. 1800 measurements were averaged for each result and the Blackman-Harris 3-term apodisation method [37] was used to remove spectral artifacts which are not related to sample absorption.

\section{Results and discussion}

\subsection{Scanning electron microscopy (SEM) characterization}

Before presenting the THz measurements, the results of structural studies via SEM are shown to confirm pellet composition and morphology. Particle size and shape of the starting powders are two important parameters that need to be characterized in order to estimate the importance of scattering. In addition, one must check to ensure that the assumptions of MG theory are consistent with the morphology of the powder particles. 

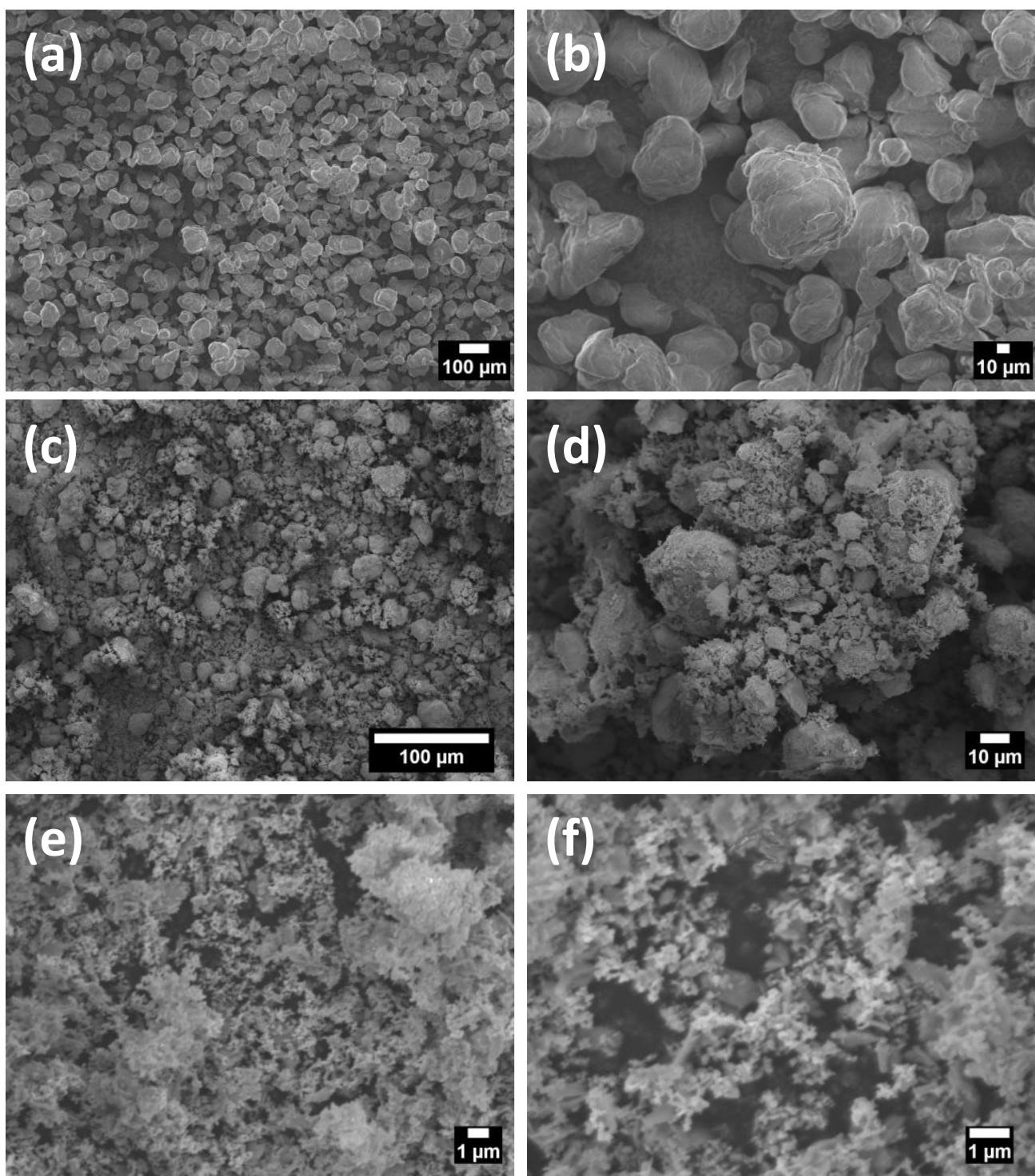

Figure 1. SEM images of the PE ( $\mathrm{a}$ and $\mathrm{b}$ ), $\mathrm{CuO}$ ( $\mathrm{c}$ and $\mathrm{d}$ ) and $\mathrm{ZnO}$ (e and f) unconsolidated powders at various magnifications.

SEM images corresponding to PE and the two initial oxide powders are shown in Fig. 1. The initial particle shape in all cases is roughly spherical with diameter less than $100 \mu \mathrm{m}$. Unconsolidated PE [Fig. 1(a) and (b)] are the largest particles, though once the pellets are consolidated, we expect the PE particles to deform and provide an approximately uniform (though porous) matrix. Figures 1(c) and (d) show the morphology of the $\mathrm{CuO}$ particles. Most of the $\mathrm{CuO}$ particles are smaller than $50 \mu \mathrm{m}$ in diameter. Note that the particle shapes are nonspherical and there are some porous aggregates of particles. This morphology appears to originate from sintering. Despite the aspherical shape of the particles, we nonetheless assume the validity of MG theory due to its isotropic distribution. Finally, in Fig. 1(e) and (f), we see that $\mathrm{ZnO}$ particles are even smaller than the $\mathrm{CuO}$ particles. The particle size is in the nanometric range with large irregular aggregates in most cases smaller than $10 \mu \mathrm{m}$. Despite 
the fact that the nanometric particles appear to be spherical, the particle aggregate sizes evident in the electron micrographs appear to rather disperse. This may impact the validity of $\mathrm{MG}$ theory for the $\mathrm{ZnO}$ oxide.
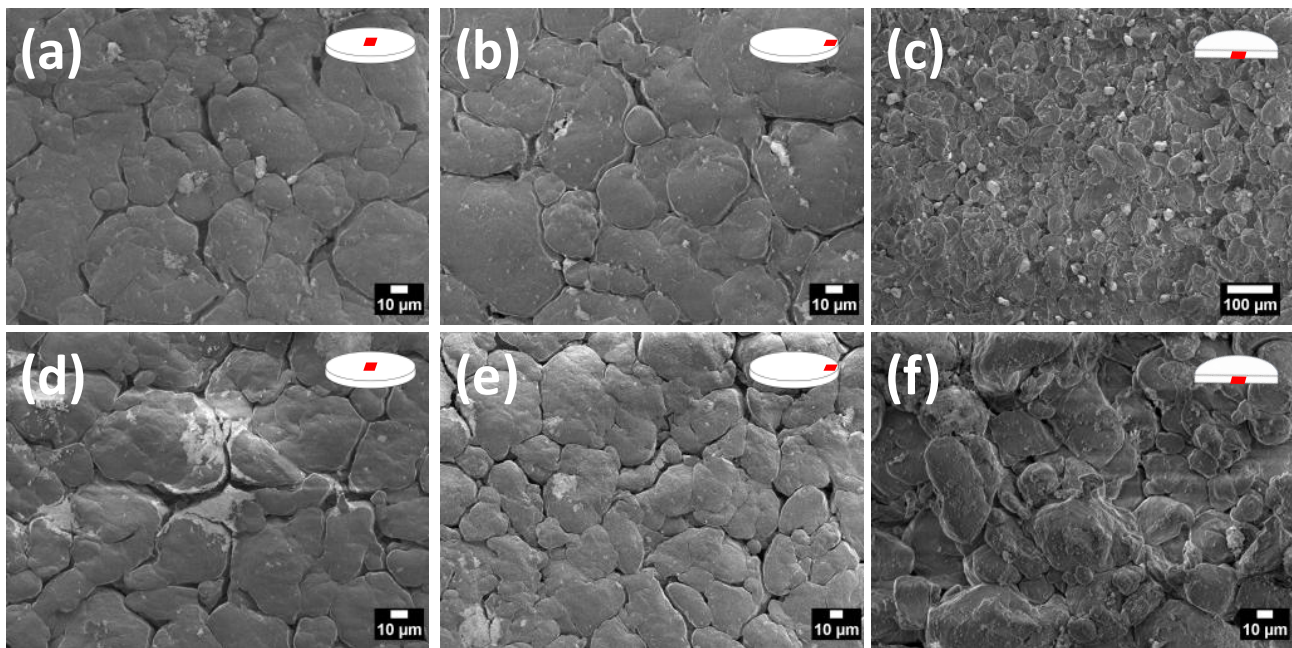

Figure 2. SEM images of the pressed $\mathrm{CuO}_{-} 10$ pellet in the center (a) and the edge (b) of the pellet surface, and on the cross section on a cut through the pellet (c). ZnO_10 pellet SEM images in the center (d) and the edge (e) of the pellet surface, and the cross section on a cut through the pellet (f).

The fabricated pellets were also observed by SEM to ensure uniformity of the particle distribution within the PE pellet. Figures 2(a)-(c) show SEM images obtained on the CuO_10 pellet. The images appear to be qualitatively consistent with the calculated porosities in Table I. Moreover, the distribution of $\mathrm{CuO}$ particles is fairly homogeneous, both on the surface and in the cross section through the pellet. On the other hand, Figures 2(d)-(f) show the dispersion of the oxide in the $\mathrm{ZnO}_{-} 10$ pellet. Despite the size of the aggregates is lower than $10 \mu \mathrm{m}$, here their distribution is not as regular as in the previous case. The cross section [Fig. 2(f)] suggests a high degree of homogeneity of the oxide particle distribution within the pellet.
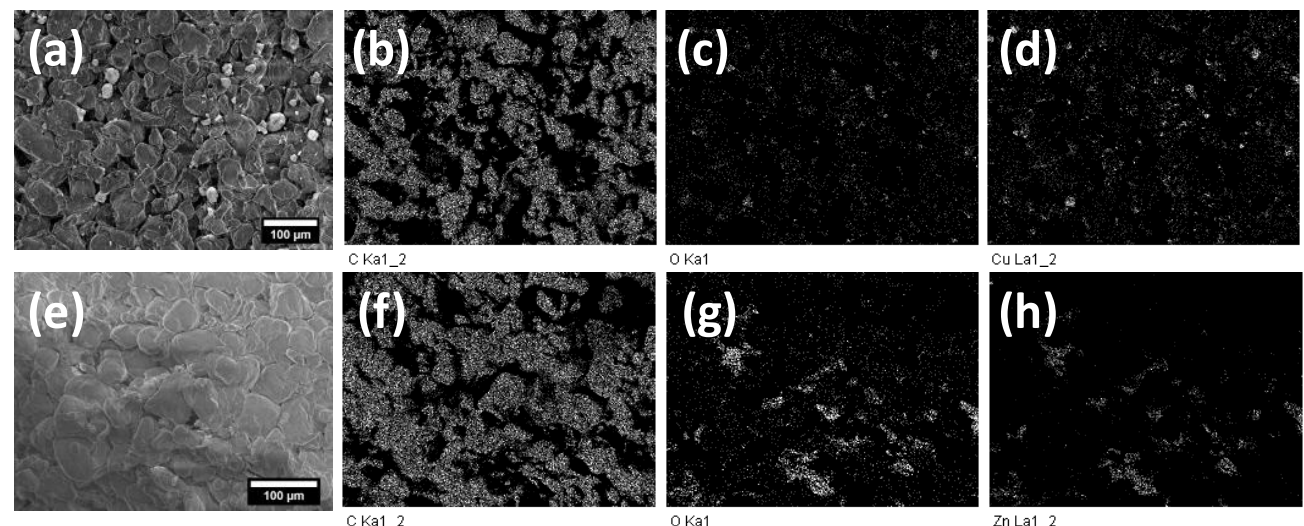

Figure 3. Cross-section SEM and EDS images. CuO_10 pellet: (a) SEM, (b) EDS map of C distribution, (c) EDS map of $\mathrm{O}$ distribution, and (d) EDS map of $\mathrm{Cu}$ distribution; ZnO_10.0 pellet: (e) SEM, (f) EDS map of C distribution, (g) EDS map of O distribution, and (h) EDS map of Cu distribution. EDS maps have the same magnifications as their corresponding SEM image. 
Finally, energy dispersive x-ray analysis (EDS) has been used to provide chemical information concerning of the particle $(\mathrm{C}, \mathrm{O}, \mathrm{Cu}, \mathrm{Zn})$ distribution. Fig. 3(a)-(d) provide the results for the $\mathrm{CuO} \_10$ pellet. We observe substantial homogeneity of the $\mathrm{C}$ distribution, originating in the $\mathrm{PE}$. The $\mathrm{O}$ distribution reflects both the $\mathrm{ZnO}$ and $\mathrm{CuO}$ particles, as well as air trapped in the pores; the $\mathrm{Cu}$ concentration originates from the $\mathrm{CuO}$ particles. In all cases, a high degree of homogeneity is observed. A similar analysis for the $\mathrm{ZnO}_{-} 10.0$ sample is shown in Fig. 3(e)-(h). Overall, the homogeneity is high, though we note features consistent with some small-scale aggregation of oxide particles observed by SEM.

We therefore conclude that homogeneity of the oxide particle dispersion in PE is sufficiently high so that measurements obtained from a specific point on the pellet (constrained by the sample holder) are representative of the entire pellet.

\subsection{Terahertz characterization}

The THz pulse transmitted through pellets listed in Table 1 was measured following the procedure discussed in Sec. 3. Figure 4 shows the THz signal (time-dependent electric field) obtained for each weight filling factor $f_{w}$ for both $\mathrm{CuO}$ and $\mathrm{ZnO}$. Note that the reference signal (obtained with the pellet removed from the sample holder) is the one with the highest amplitude and smallest optical delay.

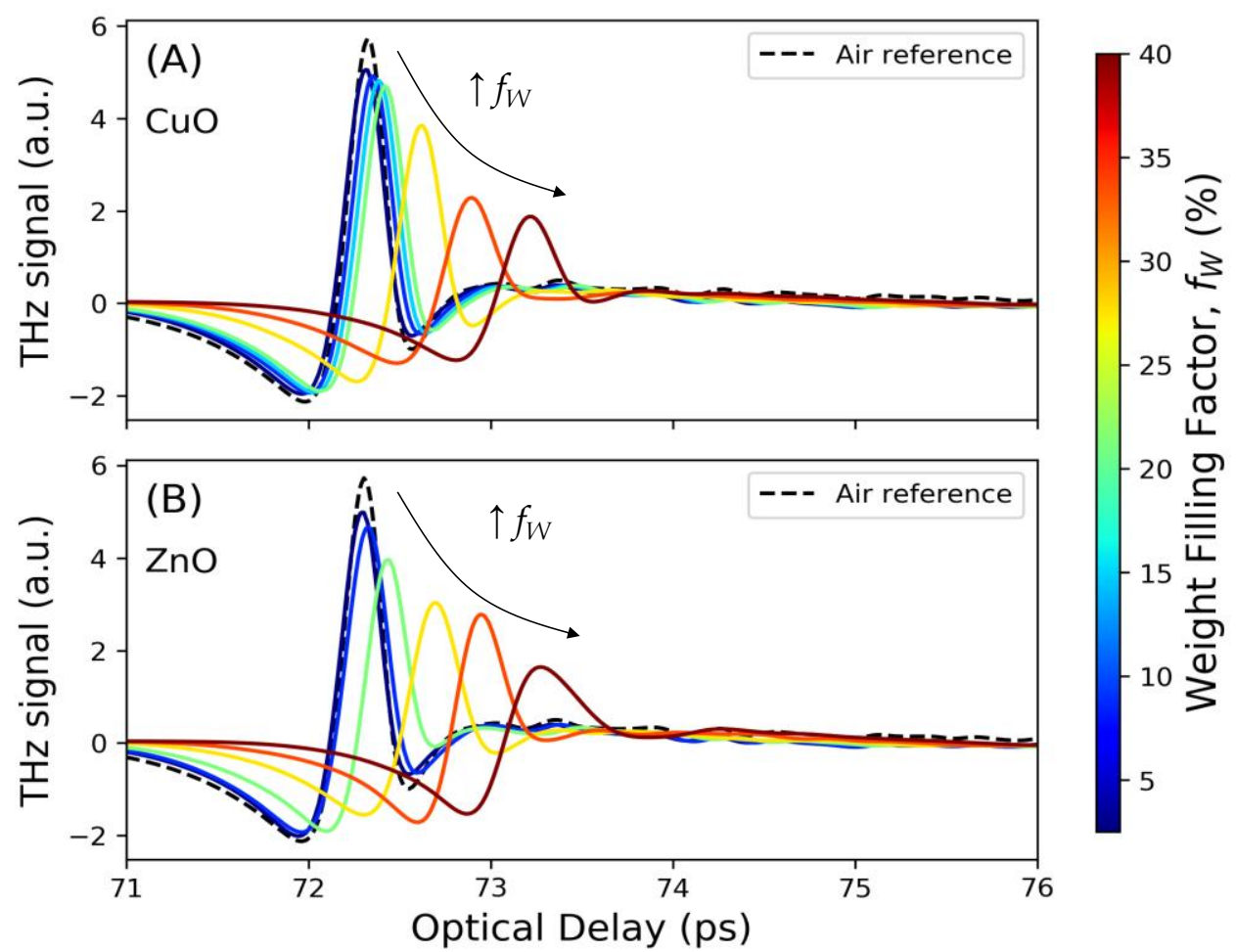

Figure 4. THz signals of (a) $\mathrm{CuO}$ and (b) $\mathrm{ZnO}$ particles dispersed in PE pellets for various weight filling factors $f_{w}$.

Not surprisingly, the amplitude of the $\mathrm{THz}$ pulse decreases as the oxide-particle weight filling factor $f_{w}$ increases indicating attenuation as well as increased surface reflections associated with the Fresnel coefficient; there is also a larger overall time delay for higher $f_{w}$, indicating an increased refractive index. Pellets containing either oxide powder are less transmissive than pure PE, as expected. 
The frequency dependence of the transmitted THz pulses is shown in Fig. 5.

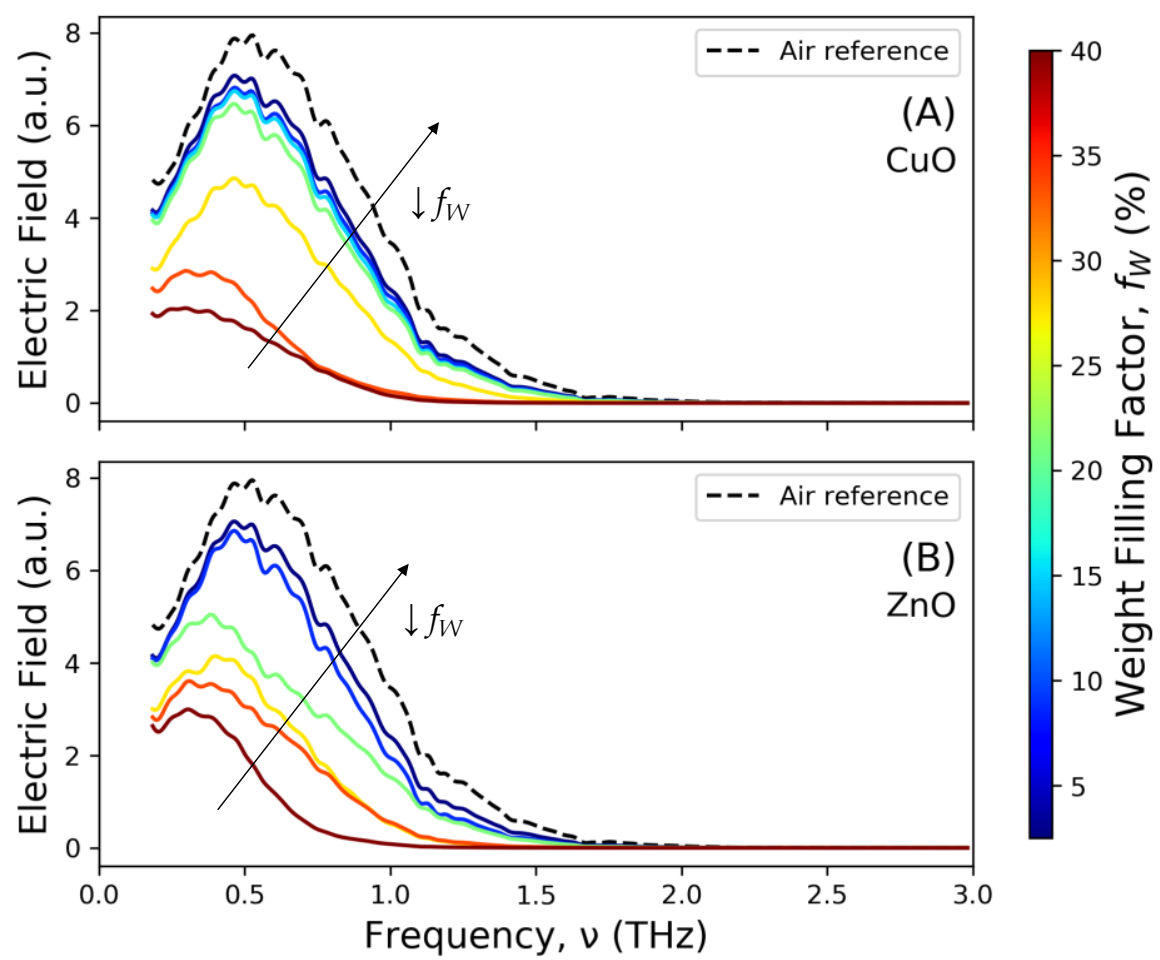

Figure 5. Frequency dependence power spectrum of the transmitted $\mathrm{THz}$ pulses for (a) $\mathrm{CuO}$ and (b) $\mathrm{ZnO}$ powders in PE pellets for various $f_{w}$.

The spectral amplitude of the signal is reduced at all frequencies as $f_{w}$ increases. Note that no pronounced absorption lines are detected for any of the pellets in this frequency range as the lowest far-infrared-active modes in $\mathrm{CuO}$ are at and above $12.96 \mathrm{THz}$ [38] while those in $\mathrm{ZnO}$ are at or above $13.11 \mathrm{THz}[39,40]$. THz absorption features in $\mathrm{PE}$ are expected to be spectrally diffuse as polymers rarely show pronounced features in the measurement bandwidth.

The optical constants were extracted from these data. From Eqs. (2) and (3), the refractive index $n(v)$ and the absorption coefficient $\alpha(v)$ were obtained for the pellet as shown in Fig. 6. In the following, the term effective is used to describe the apparent optical constants for the pellets (in contrast to the extracted values for the oxides). 
(a)
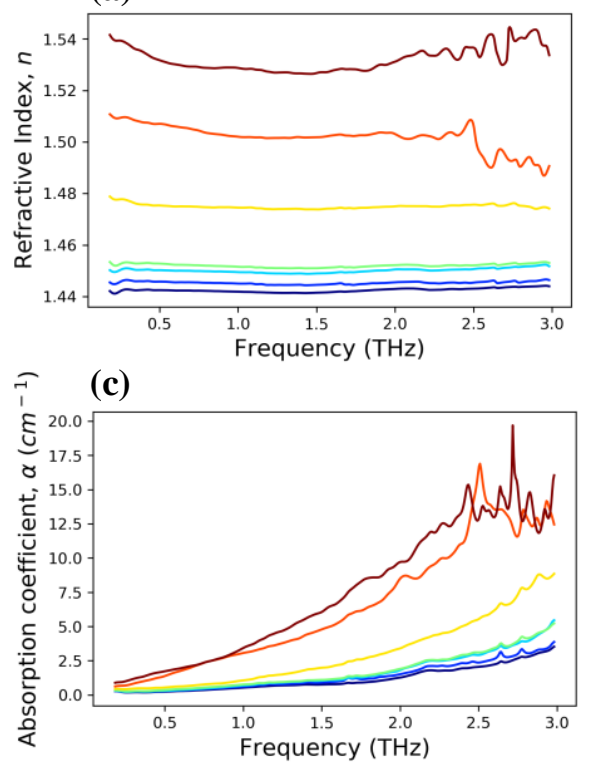

(b)

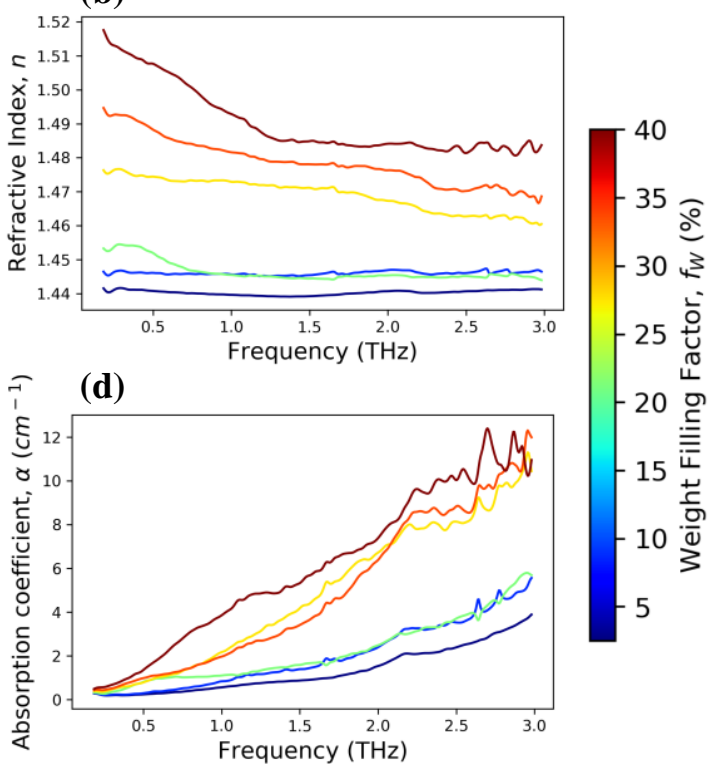

Figure 6. Effective optical constants for the pellets directly obtained from experimental data. Effective refractive index $n$ for (a) $\mathrm{CuO}$ and (b) $\mathrm{ZnO}$ pellets, and effective absorption coefficient $\alpha$ for (c) $\mathrm{CuO}$ and (d) $\mathrm{ZnO}$ pellets with various weight filling factors $f_{w}$.

Figures 6(a) and (b) reveal refractive indices $n(v)$ that are spectrally flat within the experimental bandwidth. On the other hand, Figs. 6(c) and (d) show a characteristic powerlaw increase of the absorption coefficient $\alpha(v)$ with frequency $[15,16]$. We also observe that $\mathrm{CuO}$ seems to exhibit slightly higher values for $n(v)$ and $\alpha(v)$ than $\mathrm{ZnO}$. Of note, features observed above $\sim 2 \mathrm{THz}$ are attributed largely to the low SNR. The effective optical constants $\varepsilon_{r}$ ' and $\varepsilon_{r}$ "' are computed using Eqs. (5) and (6), and shown in Fig. 7. The dispersion in $\varepsilon_{r}$ ' for both materials is negligible (as for $n$ values), and increases with the $f_{w} \cdot \varepsilon_{r}$ " increases by a power law with $v$, reflecting similar behavior for $\alpha(v)$.

MG theory [Eq. (8)] is then used to extract, for each $f_{W}$, optical constants of the oxides from the effective optical constants for the pellets. For each $f_{W}$, the corresponding oxide optical constants are calculated by computing the complex permittivity, and the obtained mean value and its standard deviation, averaged over all filling factors of different $f_{W}$, are plotted in the top panels of Figs. 7(a)-(d). 
(a)

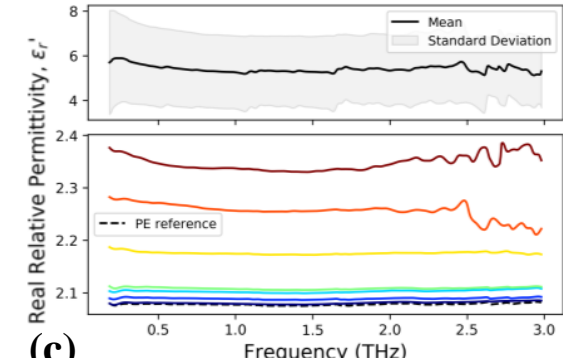

(c)

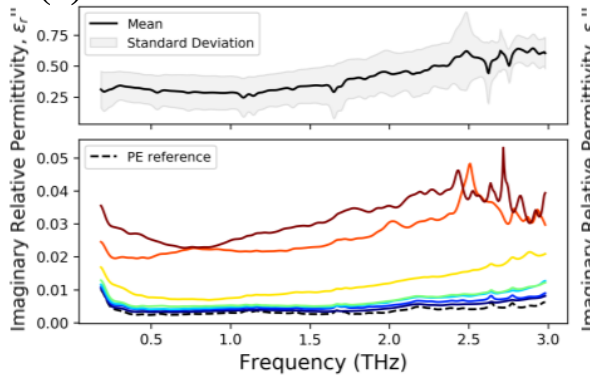

(b)

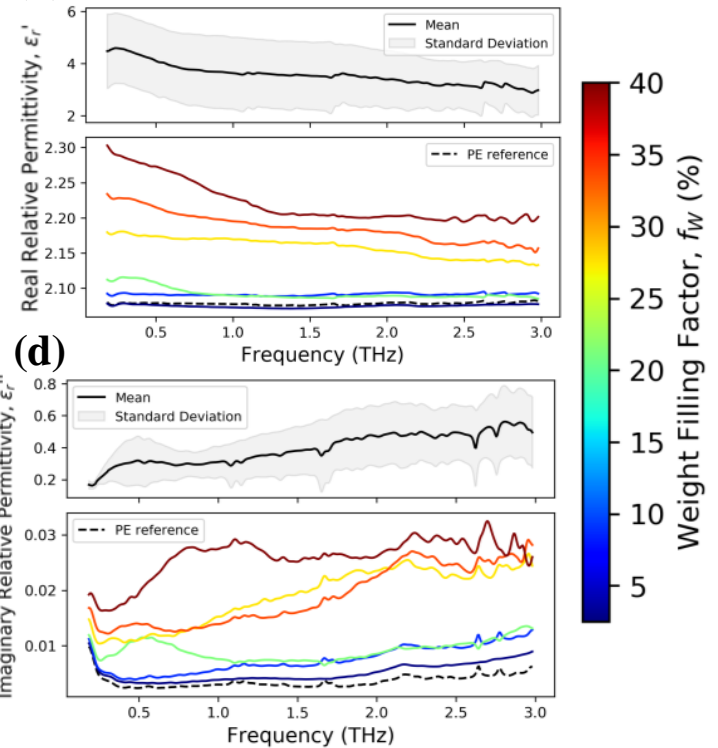

Figure 7. $\varepsilon_{r}$ ' for (a) $\mathrm{CuO}$ and (b) $\mathrm{ZnO}$ pellets, $\varepsilon_{r}$ "' for (c) $\mathrm{CuO}$ and (d) $\mathrm{ZnO}$ pellets for various $f_{w}$. In the top frame of each panel the properties of the inclusion oxides are shown after being calculated by MG theory. The standard deviation of mean values is represented by the shaded grey area in the top panels. Note that these results do not account for the presence of air associated with the $15 \%$ measured pellet porosity.

As is reported in Table 1 (porosity, $P$ ) and discussed in Sec. 4.1 these pellets contain trapped air ( $\sim 15 \%$ in volume) that has not been considered in this calculation. The effect of $P$ on the optical measured properties has been previously discussed elsewhere [41]. Parrot $e t$ al. have shown that there may be considerable differences in the extracted properties depending if porosity $P$ is considered or not when applying the MG model. In this work, differences of around a $5 \%$ in the refractive index $n$ are measured in samples with $P$ values of 8 and $9 \%$. When the calculation takes into account the pellet porosity, the obtained parameters tend to increase because the volume occupied by air is non-absorbing. 
(a)
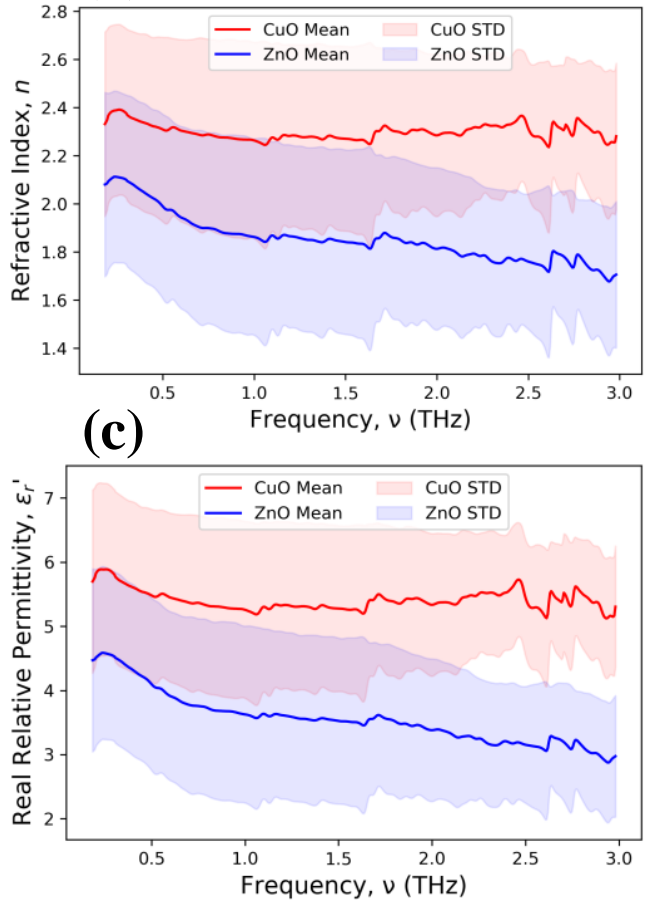

(b)

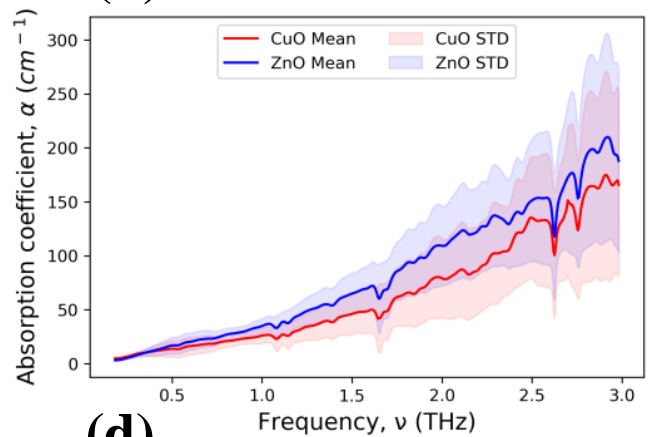

(d)

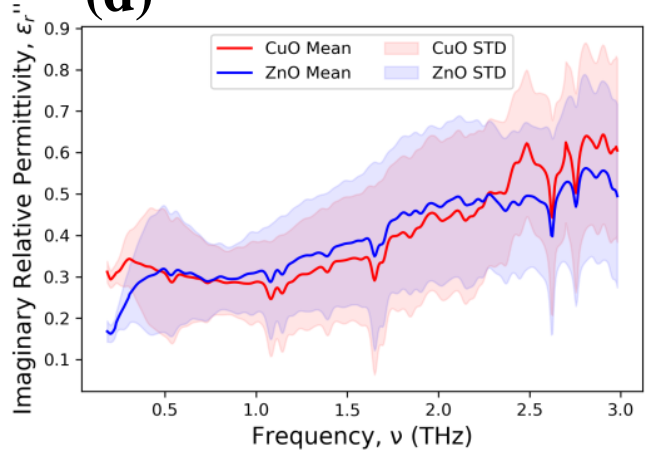

Figure 8. $\mathrm{CuO}$ and $\mathrm{ZnO}$ optical properties between 0.18 and $3 \mathrm{THz}$ : (a) refractive index, (b) absorption coefficient, (c) real relative permittivity $\varepsilon^{\prime}$, and (d) imaginary relative permittivity $\varepsilon^{\prime}$. All the represented optical properties correspond to the oxide inclusions.

From Fig. 8 one sees that $\varepsilon_{r}$ ' is larger for $\mathrm{CuO}$ than for $\mathrm{ZnO}$; meanwhile $\mathrm{ZnO}$ presents higher values of $\varepsilon_{r}$ "'. Moreover, as stated before, these values slightly underestimate the permittivity values of the inclusions due to the air effect.

Table 2. Inclusions' mean real permittivity $\varepsilon_{r}$ ' and refractive index $n$ calculated by the MG model.

\begin{tabular}{ccc} 
Material & $\boldsymbol{\varepsilon}_{\mathbf{r}}{ }^{,}$ & $\mathbf{n}$ \\
\hline $\mathrm{CuO}$ & 5.41 & 2.30 \\
$\mathrm{ZnO}$ & 3.55 & 1.85
\end{tabular}

In Table 2 are shown the mean values of real permittivity $\varepsilon_{r}{ }^{\prime}$ and refractive index $n$ for both materials along the measured frequency range. These optical constants may depend on particle shape or size, but are insensitive to frequency $v$.

Both $\mathrm{CuO}$ and $\mathrm{ZnO}$ are anisotropic crystals, which means that the dielectric properties must be described by tensors, and a number of measurements of the optical constants in the $\mathrm{THz}$ regime have been made on single crystals of these materials [42-46]. Our samples, though, have a high degree of isotropy at the macroscopic level since the oxide particles are randomly distributed and oriented in the pellets - electron micrographs do not show any obvious orientation of particles due to pressing - leading to an absence of birefringence. 
$\mathrm{CuO}$ is monoclinic. In Ref. [45] is reported room-temperature ordinary and extraordinary refractive indices $\sim 3$ with birefringence $\lesssim 2 \%$ with total dispersion $\lesssim 2 \%$ in the range $\sim 500$ GHz-2 THz. Dispersion tends to higher values of refractive index with increasing frequency, in contrast with our inferred behavior [Fig. 8(a)], but in all cases dispersion is relatively weak. Reported refractive indices $[45,46]$ tend to be larger than our extracted value $(2.3$, see Table 2 ), but if considering porosity, the inferred values from our work may be somewhat higher, but likely not enough to account for the discrepancies. In any case, birefringence in single crystals is small, so that our neglect of anisotropy of the underlying crystals does not appear to be able to account for the discrepancies either.

In recent studies on $\mathrm{CuO}$ films, Alqahtani et al. [47] obtained values of $n$ around 1.5; meanwhile Dolai et al. [48] found values around 3.5 and $\varepsilon_{r}$ ' close to 11. Clearly, there is a large range of values reported for various types of samples, and morphology as well as the method of parameter extraction surely play a role in contributing to this range.

The absorption coefficients in Refs. [45] appear to obey Jonscher's universal power law (otherwise known as the universal dielectric response; see below). They report a similar frequency dependence of the absorption coefficient for $\mathrm{CuO}$ to the one we have, reaching values around $25 \mathrm{~cm}^{-1}$ at $1 \mathrm{THz}$.

$\mathrm{ZnO}$ has a hexagonal wurtzite structure. Fujii et al. [44] report $\varepsilon_{r}{ }^{\prime} \sim 8$, and in Azad et al. [43] $n \sim 2.8$. The room-temperature ordinary and extraordinary refractive indices of $\mathrm{ZnO}$ in the THz range have been measured to be 2.67 and 2.84 , respectively, from $180 \mathrm{GHz}$ to $1.25 \mathrm{THz}$ with little dispersion [42]. There appears thus to be a discrepancy between these reported values and ours (Table 2). The absorption coefficient of $\mathrm{ZnO}$ measured in Ref. [42] also obeys Jonscher's law, but overall with considerably lower absorption. Again, scattering as well as the different crystal structure may play a role in the differences in the values.

In Ref. [42], however, reported values of $\alpha$ are almost an order of magnitude lower than ours. The role of scattering in our experiment due to the intrinsically inhomogeneous nature of particles pressed into pellets may account for the much higher value of attenuation we infer for $\mathrm{ZnO}$.

Another possible source of the differences between the values we infer from the pressed pellets and measurements made on single crystals may stem from differences in the optical constants of the particles themselves with respect to bulk. Onwudiwe et al. [49] and Rehman et al. [50] both reported $\varepsilon_{r}{ }^{\prime}$ around 3.5 in the $\mathrm{MHz}$ and $\mathrm{GHz}$ ranges for $\mathrm{ZnO}$ nano- and microspheres, respectively, which is close to the value we infer from the pressed pellets. Measurements of particles well outside our frequency range exist [51] though comparisons with our work may have limited meaning.

Finally, based on $\varepsilon_{r}$ "' and Eq. (7), the conductivity of each material is calculated. Figure 9 illustrates the results for each oxide. The experimental data has been fitted with the Jonscher's universal power law, widely used for fitting frequency dependence of conductivity [52]

$$
\sigma_{A C}=\sigma_{0}+A \omega^{n},
$$

where $\sigma_{0}$ is the zero-frequency electrical conductivity, and the pre-factor $A$ (not electric-field amplitude here) and exponent $n$ (not refractive index here) depend on the material and temperature. The value of $n$ is smaller than unity when the conductivity is dominated by the 
long-range movement of charge carriers; meanwhile it is larger than 1 when short-range hopping dominates transport [53-56].

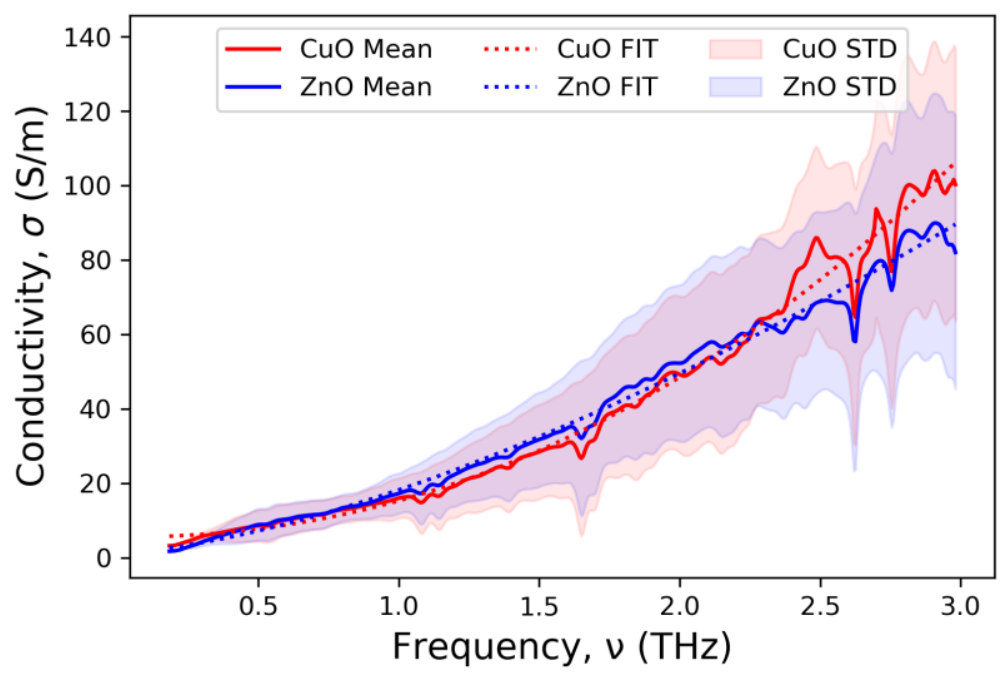

Figure 9. Oxide conductivity $\sigma$ inferred from pressed-pellet samples. Thick lines represent the average values, shaded regions indicate the standard deviation, and dashed lines show the fit to Jonscher's model for each oxide.

We observe a clear power-law dependence of the conductivity with $v$ and that Jonscher's model fits well with the experimental data. As we expect following our observations concerning $\alpha, \mathrm{ZnO}$ has slightly higher conductivity than $\mathrm{CuO}$ until approximately $2.3 \mathrm{THz}$. Best-fit values for each measurement are presented in Table 3.

Table 3. Fitting parameters to Jonscher's law.

$\begin{array}{cccc}\text { Material } & \boldsymbol{\sigma}_{\mathbf{0}}(\mathbf{S} / \mathbf{m}) & \boldsymbol{A} & \boldsymbol{n} \\ \mathrm{CuO} & 9.26 & 17.0 & 2.10 \\ \mathrm{ZnO} & 4.66 & 28.7 & 1.47\end{array}$

Last, a consistency check of MG theory is presented for both materials in Fig. 10. Here all the effective real permittivity $\varepsilon_{r}$ ' measured values are represented as a function of its corresponding volume filling factor $f_{V}$. Then, the theoretically expected effective $\varepsilon_{r}$ ' values have been calculated along the whole $f_{V}$ range by means of the MG model. We note a significant agreement between the experimental values and MG theory. 
(a)

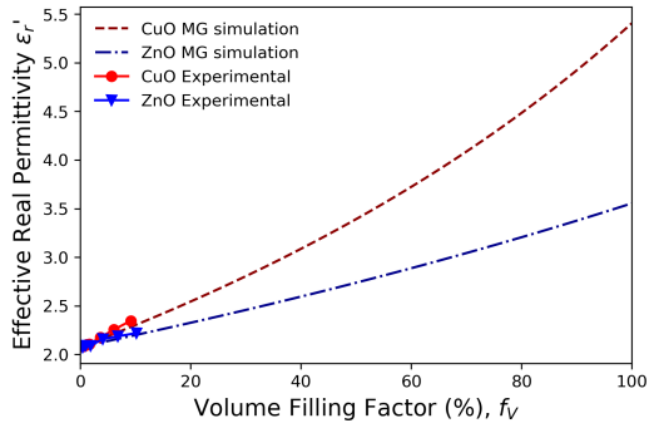

(b)

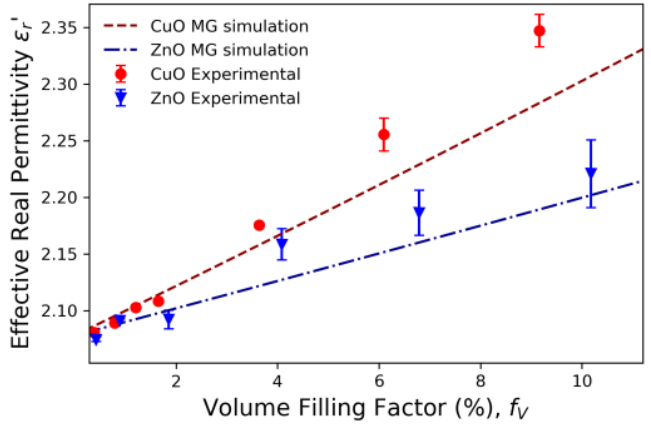

Figure 10. Comparison between experimentally measured effective permittivity values for different $f_{v}$ and MG theory for the entire range of $f_{v}$ (a), and a zoomed image around the experimental values studied here (b).

Equation (8) has been used in order to obtain these fits by considering an average PE real permittivity of 2.08, and $\mathrm{CuO}$ or $\mathrm{ZnO}$ inclusions, giving the parameters of Table 2. In Fig. 10(a) good fit between MG model and the experimental results is seen. Nevertheless, when the low-filling factor region is magnified [Fig. 10(b)], we observe slight deviations that may be due to nonsphericity of the oxide particles (which is assumed by MG theory), scattering, and uncertainties due to the presence of trapped air inside the pellets. In fact, this slight underestimation of $\varepsilon_{r}$ ' is reasonable considering that the porosity has not been taken into account in these calculations.

\section{Conclusions}

In this work the optical properties of two different metal-oxides have been characterized in the $\mathrm{THz}$ frequency range. First, pellets of $\mathrm{CuO}$ and of $\mathrm{ZnO}$ powders with various fill factors in PE matrix were pressed. The PE acts as a THz-transparent host. SEM images show a particle size distribution well below $100 \mu \mathrm{m}$. In addition, fill factors are low so diffuse scattering is likely to play only a small role. This all suggests that a static theory such as MG is appropriate. The combination of SEM and EDS has revealed a homogeneous distribution of oxide particles, with overall homogeneous and isotropic distribution.

The effective optical properties were measured in the $\mathrm{THz}$ range of a series of pellets of $\mathrm{CuO}$ or $\mathrm{ZnO}$ dispersed in PE with various oxide fill factors using THz-TDS. MG theory has been used to extracting the oxide properties from the measurements carried out on the pellets. Finally, the comparison between the experimental data and MG theory confirms the selfconsistency of the MG assumptions. Complex permittivity, refractive index, absorption coefficient and electrical conductivity coefficients have been calculated in the frequency range from $0.18 \mathrm{THz}$ to $3 \mathrm{THz}$ for each material. Results reveal that $\mathrm{CuO}$ has a higher real permittivity and refractive index, but $\mathrm{ZnO}$ has larger imaginary permittivity, absorption coefficient, and electrical conductivity. Values of $n$ inferred from our experiments are somewhat smaller than measurements on single crystals, while we infer larger values of $\alpha$ compared with published values obtained from single crystals.

While there is considerable discrepancy between our inferred values and those obtained from single crystals, there is substantially better agreement with measurements in the literature based on powders. Whether this is connected to scattering or is an intrinsic feature of small particles is to be explored in more detail. These $\mathrm{THz}$ measurements are of potential 
importance because they provide new results concerning the dielectric properties of homogeneously and isotropically distributed $\mathrm{ZnO}$ and $\mathrm{CuO}$ powders in the $100 \mathrm{GHz}$ to $3 \mathrm{THz}$ frequency range.

Acknowledgments: We gratefully acknowledge financial support from the Region of Grand Est and Institut Carnot ARTS. J. Calvo-de la Rosa acknowledges Ajuts a la Docència i a la Recerca (ADR) given by the Universitat de Barcelona and the Catalan Government for the quality accreditation given to his research group DIOPMA (2017 SGR 118).

\section{References}

[1] X. Guo, G. Zhang, Q. Li, H. Xue, H. Pang, Non-noble metal-transition metal oxide materials for electrochemical energy storage, Energy Storage Mater. 15 (2018) 171-201. doi:10.1016/j.ensm.2018.04.002.

[2] A. Dey, Semiconductor metal oxide gas sensors: A review, Mater. Sci. Eng. B Solid-State Mater. Adv. Technol. 229 (2018) 206-217. doi:10.1016/j.mseb.2017.12.036.

[3] C. V. Garcia, G.H. Shin, J.T. Kim, Metal oxide-based nanocomposites in food packaging: applications, migration, and regulations, Trends Food Sci. Technol. 82 (2018) 21-31. doi:10.1016/j.tifs.2018.09.021.

[4] F. Cao, T. Wang, X. Ji, Enhanced visible photocatalytic activity of tree-like $\mathrm{ZnO} / \mathrm{CuO}$ nanostructure on $\mathrm{Cu}$ foam, Appl. Surf. Sci. 471 (2019) 417-424. doi:10.1016/j.apsusc.2018.12.034.

[5] S. Park, S. Kim, H. Kheel, S.K. Hyun, C. Jin, C. Lee, Enhanced H2S gas sensing performance of networked $\mathrm{CuO}-\mathrm{ZnO}$ composite nanoparticle sensor, Mater. Res. Bull. 82 (2016) 130-135. doi:10.1016/j.materresbull.2016.02.011.

[6] C. Qin, Y. Wang, Y. Gong, Z. Zhang, J. Cao, CuO-ZnO hetero-junctions decorated graphitic carbon nitride hybrid nanocomposite: Hydrothermal synthesis and ethanol gas sensing application, J. Alloys Compd. 770 (2019) 972-980. doi:10.1016/j.jallcom.2018.08.205.

[7] S.E. Diltemiz, K. Ecevit, High-performance formaldehyde adsorption on $\mathrm{CuO} / \mathrm{ZnO}$ composite nanofiber coated QCM sensors, J. Alloys Compd. 783 (2019) 608-616. doi:10.1016/j.jallcom.2018.12.237.

[8] Y. Liu, Y. Chang, S. Yang, F. Li, Y. Sun, J. Wu, B. Yang, W. Cao, Improved densification behavior and energy harvesting properties of low-temperature sintered $(\mathrm{Ba}, \mathrm{Ca})(\mathrm{Zr}, \mathrm{Ti}) \mathrm{O} 3$ piezoceramics with a $\mathrm{CuO}$ additive, Ceram. Int. 45 (2019) 10518-10524. doi:10.1016/j.ceramint.2019.02.114.

[9] H. Sun, Y. Zhang, X. Liu, Y. Liu, W. Chen, Effects of $\mathrm{CuO}$ additive on structure and electrical properties of low-temperature sintered Ba0.98Ca0.02Zr0.02Ti0.98O3 lead-free ceramics, Ceram. Int. 41 (2015) 555565. doi:10.1016/j.ceramint.2014.08.104.

[10] X. Lu, L. Zhang, H. Talebinezhad, Y. Tong, Z.Y. Cheng, Effects of CuO additive on the dielectric property and energy-storage performance of $\mathrm{BaTiO} 3$-SiO 2 ceramic-glass composite, Ceram. Int. 44 (2018) 16977-16983. doi:10.1016/j.ceramint.2018.06.139.

[11] C. Barreneche, M. Martín, J. Calvo-de la Rosa, M. Majó, A. Fernández, Own-Synthetize Nanoparticles to Develop Nano-Enhanced Phase Change Materials (NEPCM) to Improve the Energy Efficiency in Buildings, Molecules. 24 (2019) 1232. doi:10.3390/molecules24071232.

[12] Y. Li, L. Zhang, L. Yu, D. Li, H. Meng, Q. Ai, J. Hu, L. Jin, J. Gao, G. Liu, Study of the structure, electrical properties, and energy storage performance of $\mathrm{ZnO}$-modified $\mathrm{Ba} 0.65 \mathrm{Sr} 0.245 \mathrm{Bi} 0.07 \mathrm{TiO} 3 \mathrm{~Pb}$-free ceramics, Ceram. Int. (2019). doi:10.1016/j.ceramint.2019.08.111.

[13] C.W. Tao, X.Y. Geng, J. Zhang, R.X. Wang, Z. Bin Gu, S.T. Zhang, Bi0.5Na0.5TiO3-BaTiO3$\mathrm{K} 0.5 \mathrm{Na} 0.5 \mathrm{NbO} 3: \mathrm{ZnO}$ relaxor ferroelectric composites with high breakdown electric field and large energy storage properties, J. Eur. Ceram. Soc. 38 (2018) 4946-4952. doi:10.1016/j.jeurceramsoc.2018.07.006.

[14] Y. Yao, Y. Li, N. Sun, J. Du, X. Li, L. Zhang, Q. Zhang, X. Hao, Enhanced dielectric and energy-storage properties in $\mathrm{ZnO}$-doped $0.9(0.94 \mathrm{Na} 0.5 \mathrm{Bi} 0.5 \mathrm{TiO} 3-0.06 \mathrm{BaTiO} 3)-0.1 \mathrm{NaNbO} 3$ ceramics, Ceram. Int. 44 (2018) 5961-5966. doi:10.1016/j.ceramint.2017.12.174.

[15] R. Hayati, A. Barzegar, Microstructure and electrical properties of lead free potassium sodium niobate piezoceramics with nano ZnO additive, Mater. Sci. Eng. B Solid-State Mater. Adv. Technol. 172 (2010) 121-126. doi:10.1016/j.mseb.2010.04.033.

[16] H.-E. Peiponen, J.A. Zeitler, Kuwata-Gonokami, A. Adibi, T. Asakura, T. Hänsch, T. Kamiya, F. Krausz, B.A.J. Monemar, H. Venghaus, H. Weber, H. Weinfurter, Terahertz spectroscopy and imaging, Springer Series in Optical Sciences The, 2013. doi:10.1007/978-3-642-28738-1.

[17] Zhiiping Jiang, Xi-Cheng Zhang, Sensing with Terahertz Radiation, 2003.

[18] M.R. Leahy-Hoppa, M.J. Fitch, X. Zheng, L.M. Hayden, R. Osiander, Wideband terahertz spectroscopy of explosives, Chem. Phys. Lett. 434 (2007) 227-230. doi:10.1016/j.cplett.2006.12.015.

[19] M.J. Fitch, M.R. Leahy-Hoppa, E.W. Ott, R. Osiander, Molecular absorption cross-section and absolute 
absorptivity in the THz frequency range for the explosives TNT, RDX, HMX, and PETN, Chem. Phys. Lett. 443 (2007) 284-288. doi:10.1016/j.cplett.2007.06.108.

[20] A.G. Davies, A.D. Burnett, W. Fan, E.H. Linfield, J.E. Cunningham, Terahertz spectroscopy of explosives and drugs, Mater. Today. 11 (2008) 18-26. doi:10.1016/S1369-7021(08)70016-6.

[21] Y. Wang, Q. Wang, Z. Zhao, A. Liu, Y. Tian, J. Qin, Rapid qualitative and quantitative analysis of chlortetracycline hydrochloride and tetracycline hydrochloride in environmental samples based on terahertz frequency-domain spectroscopy, Talanta. 190 (2018) 284-291. doi:10.1016/j.talanta.2018.08.008.

[22] T. Chen, Q. Zhang, Z. Li, X. Yin, F. Hu, Experimental and theoretical investigations of tartaric acid isomers by terahertz spectroscopy and density functional theory, Wuhan Univ. J. Nat. Sci. 11 (2018) 1633 1635. doi:10.1016/j.saa.2018.06.091.

[23] C. Du, X. Zhang, Z. Zhang, Quantitative analysis of ternary isomer mixtures of saccharide by terahertz time domain spectroscopy combined with chemometrics, Vib. Spectrosc. 100 (2019) 64-70. doi:10.1016/j.vibspec.2018.11.003.

[24] N.S. Almodovar, J. Portelles, O. Raymond, J. Heiras, J.M. Siqueiros, Characterization of the dielectric properties and alternating current conductivity of the SrBi5-xLaxTi4FeO $18(\mathrm{x}=0,0.2)$ compound, J. Appl. Phys. 102 (2007) 3-10. doi:10.1063/1.2824898.

[25] L.S. Kadyrov, E.S. Zhukova, V.I. Torgashev, B.P. Gorshunov, A.S. Prokhorov, E.A. Motovilova, F. Fischgrabe, V. Moshnyaga, T. Zhang, R. Kremer, U. Pracht, S. Zapf, J. Pokorný, G. Untereiner, S. Kamba, M. Dressel, Terahertz-infrared spectroscopy of overdoped manganites La1-xCaxMnO3, Phys. B Condens. Matter. 460 (2015) 199-201. doi:10.1016/j.physb.2014.11.070.

[26] S.B. Kang, D.C. Chung, S.J. Kim, J.K. Chung, S.Y. Park, K.C. Kim, M.H. Kwak, Terahertz characterization of Y2O3-added AlN ceramics, Appl. Surf. Sci. 388 (2016) 741-745. doi:10.1016/j.apsusc.2016.02.152.

[27] G.B. Jung, Y. Myung, Y.J. Cho, Y.J. Sohn, D.M. Jang, H.S. Kim, C.W. Lee, J. Park, I. Maeng, J.H. Son, C. Kang, Terahertz spectroscopy of nanocrystal-carbon nanotube and -graphene oxide hybrid nanostructures, J. Phys. Chem. C. 114 (2010) 11258-11265. doi:10.1021/jp1019894.

[28] A.L. Companion, Theory and Applications of Diffuse Reflectance Spectroscopy, Springer, Boston, MA, 1965. doi:doi.org/10.1007/978-1-4684-8691-9_19.

[29] J.C. Maxwell Garnett, Colours in Metal Glasses and in Metallic Films, R. Soc. 203 (1904) 385-420.

[30] J.C. Maxwell Garnett, Colours in Metal Glasses and in Metallic Films in Metallic Solutions - II, R. Soc. 205 (1905) 237-288.

[31] B. Michael, E.W. Van Stryland, D.R. Williams, W.L. Wolfe, Handbook of Optics, 2nd ed., Optical Society of America, 1995.

[32] M. Scheller, C. Jansen, M. Koch, Applications of Effective Medium Theories in the Terahertz Regime, in: Recent Opt. Photonic Technol., 2010: p. 450 pp. doi:6533.

[33] V.A. Markel, Introduction to the Maxwell Garnett approximation: tutorial, J. Opt. Soc. Am. A. 33 (2016) 1244. doi:10.1364/JOSAA.33.001244.

[34] L. Jylhä, A. Sihvola, Equation for the effective permittivity of particle-filled composites for material design applications, J. Phys. D. Appl. Phys. 40 (2007) 4966-4973. doi:10.1088/0022-3727/40/16/032.

[35] M.Y. Koledintseva, R.E. Dubroff, R.W. Schwartz, A Maxwell Garnett model for dielectric mixtures containing conducting particles at optical frequencies, Prog. Electromagn. Res. 63 (2006) 223-242. doi:10.2528/PIER06052601.

[36] H. Namkung, J. Kim, H. Chung, M.A. Arnold, Impact of pellet thickness on quantitative terahertz spectroscopy of solid samples in a polyethylene matrix, Anal. Chem. 85 (2013) 3674-3681. doi:10.1021/ac302017d.

[37] T. Lo, I.S. Gregory, C. Baker, P.F. Taday, W.R. Tribe, M.C. Kemp, The very far-infrared spectra of energetic materials and possible confusion materials using terahertz pulsed spectroscopy, Vib. Spectrosc. 42 (2006) 243-248. doi:10.1016/j.vibspec.2006.03.002.

[38] M. Balık, V. Bulut, I.Y. Erdogan, Optical, structural and phase transition properties of $\mathrm{Cu} 2 \mathrm{O}, \mathrm{CuO}$ and $\mathrm{Cu}$ 2O/CuO: Their photoelectrochemical sensor applications, Int. J. Hydrogen Energy. (2018) 1-12. doi:10.1016/j.ijhydene.2018.08.159.

[39] R. Udayabhaskar, B. Karthikeyan, Optical and phonon properties of $\mathrm{ZnO}: \mathrm{CuO}$ mixed nanocomposite, J. Appl. Phys. 115 (2014). doi:10.1063/1.4870447.

[40] M. Sathya, K. Pushpanathan, Synthesis and Optical Properties of Pb Doped ZnO Nanoparticles, Appl. Surf. Sci. 449 (2018) 346-357. doi:10.1016/j.apsusc.2017.11.127.

[41] E.P. Parrott, J.A. Zeitler, L.F. Gladden, Accurate determination of optical coefficients from chemical samples using terahertz time-domain spectroscopy and effective medium theory, Opt. Lett. 34 (2009) 3722. doi:10.1364/OL.34.003722.

[42] Y. Kim, J. Ahn, B.G. Kim, D.S. Yee, Terahertz birefringence in zinc oxide, Jpn. J. Appl. Phys. 50 (2011). doi:10.1143/JJAP.50.030203.

[43] A.K. Azad, J. Han, W. Zhang, Terahertz dielectric properties of high-resistivity single-crystal ZnO, Appl. Phys. Lett. 88 (2006) 1-3. doi:10.1063/1.2164903.

[44] T. Fujii, A. Ando, Y. Sakabe, Characterization of dielectric properties of oxide materials in frequency range from GHz to THz, J. Eur. Ceram. Soc. 26 (2006) 1857-1860. doi:10.1016/j.jeurceramsoc.2005.09.094. 
[45] S.P.P. Jones, S.M. Gaw, K.I. Doig, D. Prabhakaran, E.M.H. Wheeler, A.T. Boothroyd, J. Lloyd-Hughes, High-temperature electromagnons in the magnetically induced multiferroic cupric oxide driven by intersublattice exchange, Nat. Commun. 5 (2014). doi:10.1038/ncomms4787.

[46] C.D.W. Mosley, M. Failla, D. Prabhakaran, J. Lloyd-Hughes, Terahertz spectroscopy of anisotropic materials using beams with rotatable polarization, Sci. Rep. 7 (2017) 1-9. doi:10.1038/s41598-017-125680 .

[47] M.S. Alqahtani, N.M.A. Hadia, S.H. Mohamed, Effect of oxidation time on structural, optical and electrical properties of mixed copper oxides nanocrystallites, Optik (Stuttg). 173 (2018) 101-109. doi:10.1016/j.ijleo.2018.08.016.

[48] S. Dolai, R. Dey, S. Das, S. Hussain, R. Bhar, A.K. Pal, Cupric oxide $(\mathrm{CuO})$ thin films prepared by reactive d.c. magnetron sputtering technique for photovoltaic application, J. Alloys Compd. 724 (2017) 456-464. doi:10.1016/j.jallcom.2017.07.061.

[49] D.C. Onwudiwe, T. Arfin, C.A. Strydom, Surfactant mediated synthesis of $\mathrm{ZnO}$ nanospheres at elevated temperature, and their dielectric properties, Superlattices Microstruct. 81 (2015) 215-225. doi:10.1016/j.spmi.2015.02.003.

[50] S. ur Rehman, J. Liu, R. Ahmed, H. Bi, Synthesis of composite of $\mathrm{ZnO}$ spheres with polyaniline and their microwave absorption properties, J. Saudi Chem. Soc. (2018) 0-6. doi:10.1016/j.jscs.2018.04.006.

[51] P. Vincent, D.K. Kim, J.H. Kwon, J.H. Bae, H. Kim, Correlating the nanoparticle size dependent refractive index of $\mathrm{ZnO}$ optical spacer layer and the efficiency of hybrid solar cell through optical modelling, Thin Solid Films. 660 (2018) 558-563. doi:10.1016/j.tsf.2018.06.004.

[52] A.K. Jonscher, The 'universal' dielectric response, Naturevolume. 267 (1977) 673-679. doi:10.1038/267673a0.

[53] K. Funke, Jump relaxation in solid electrolytes, Prog. Solid State Chem. 22 (1993) 111-195. doi:10.1016/0079-6786(93)90002-9.

[54] L. Biswal, P.R. Das, B. Behera, Frequency dependent electrical properties of Na2Pb2R2W2Ti4Nb4O30(R $=$ Nd, Sm) ceramics, J. Adv. Ceram. 3 (2014) 215-223. doi:10.1007/s40145-014-0112-2.

[55] S. Sen, R.N.P. Choudhary, Impedance studies of Sr modified BaZr 0.5 Ti 0.95 O 3 ceramics, Mater. Chem. Phys. 87 (2004) 256-263. doi:10.1016/j.matchemphys.2004.03.005.

[56] M. Vijayakumar, S. Selvasekarapandian, M.S. Bhuvaneswari, G. HiranKumar, G. Ramprasad, R. Subramanian, P.C. Angelo, Synthesis and ion dynamics studies of nanocrystalline Mg stabilized zirconia, Phys. B Condens. Matter. 334 (2003) 390-397. doi:10.1016/S0921-4526(03)00103-0. 